\title{
Analysis of Microfluidic Pipes in a W-band Waveguide for Determining Complex Permittivity.
}

\author{
Robert C. Jones \\ EECS. Queen Mary University of \\ London \\ London, UK \\ r.c.jones@qmul.ac.uk \\ Rostyslav Dubrovka \\ EECS. Queen Mary University of \\ London \\ London, UK \\ r.dubrovka@qmul.ac.uk
}

\author{
Andre Sarker Andy \\ EECS. Queen Mary University of \\ London \\ London, UK \\ a.andy@qmul.ac.uk \\ Akram Alomainy \\ EECS. Queen Mary University of \\ London \\ London, UK \\ a.alomainy@qmul.ac.uk
}

\author{
Robert Donnan \\ EECS. Queen Mary University of \\ London \\ London, UK \\ r.donnan@qmul.ac.uk
}

\begin{abstract}
Determination of complex permittivity of biochemical fluid samples have been numerically investigated. The samples have been placed within a rectangular $W$-band waveguide enclosed in nanolitre volume microfluidic pipes. The effect on the $\mathrm{S}$-parameters is observed by changing the shape, diameter, depth, and complex permittivity of the pipe inside the waveguide. Pipe depth and pipe diameter has a direct influence on the drop in transmission coefficient $S_{21}$. The system shows the viability of measurements of liquid samples inside a waveguide at these frequencies. Extraction of the permittivity is not covered in this paper, however matching of experimental results to empirical dependences obtained by full wave electromagnetic solver is one method which can be used.
\end{abstract}

Keywords-Microfluidics, Waveguide, Sensing

\section{INTRODUCTION}

Many methods for microfluidic liquid sensing exist in the microwave domain, broadband measurements are possible using Quasi-Optical (QO) systems or THz-Time Domain Spectrometers, where a liquid cell with low loss windows can be used for holding liquid samples in the beampath $[1,2]$. This method is reliant on skilled loading of the liquid sample holder error due to air gaps, and evaporation of the liquid [3]. Limitations exist for QO methods, where small volumes of liquid are not measured easily due to the diffraction limit restricting the smallest beam spot size.

In literature, for waveguide based methods, most of the work focuses primarily on resonant techniques, such as ridgegap waveguides, whispering gallery modes, photonic crystal resonators, filter based waveguide methods, and waveguide slot antenna based sensors $[4,5,6,7,8]$. These methods are all inherently narrowband and hence limit extraction of permittivity to a single frequency. Non-resonant work has been found in [9] up to Ka-band.

We propose an approach where a microfluidic pipe is inserted inside a standard W-band waveguide, with a slot cut out of the broad wall, to allow for a microfluidic chamber to be placed inside. This proposed design would potentially enable measurement of nanolitre volumes of liquid samples. Microfluidics, in general, are not measured inside rectangular waveguides. It is important to understand how the dimensions of the microfluidics influence the results. Hence this paper focuses on sensitivity analysis of the microfluidic pipes to determine the feasibility of such a design at W-band.

\section{DESIGN OF THE MiCROFLUIDIC INSERT AND WAVEGUIDE}

For the presented study, W-band waveguide has been chosen $(75-110 \mathrm{GHz})$ with internal wall dimensions $2.54 \times 1.27$ $\mathrm{mm}$. At $75 \mathrm{GHz}$ a guided wavelength in the waveguide is $\lambda_{g}=6.5 \mathrm{~mm}$. For the given wavelength a waveguide length has to be set in a such way that it ensures that the distance between the discontinuity caused by the sample holder, and a waveguide port is at least $2 \lambda_{g}$. It allows the evanescent modes to decay, hence only the dominant mode $\mathrm{TE}_{10}$ is received.

The plastic chamber, in future work is to be printed using the Nanoscribe 3D printer. For this, the material IP-S will be used, the permittivity of which is calculated from measured data in [10]. The permittivity at $92.5 \mathrm{GHz}$ is $\varepsilon_{r}{ }^{\prime}=2.652$ and $\tan \delta=0.00028$.

To investigate the influence of pipe shape, a U-shaped pipe, and a W-shaped pipe are modelled as shown in Fig. 1 a) and $b$ ). The dimensions of the insert were designed with the intention for a single pipe to fit inside. The maximum pipe diameters $\left(D_{p}\right)$ used are $0.4 \mathrm{~mm}$ for the U-pipe, and $0.35 \mathrm{~mm}$ for the $\mathrm{W}$-pipe. The chamber dimensions presented in this work are length $l=3.8 \mathrm{~mm}$, width $w=0.6 \mathrm{~mm}$. The height $h=2.27 \mathrm{~mm}$ is set such that the height of the W-band waveguide is completely filled with the insert, and $1 \mathrm{~mm}$ of extra material protrudes above the slot for access to the insert. These dimensions allow for a minimum of $0.1 \mathrm{~mm}$ material surrounding the pipe.

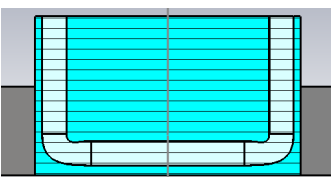

a)

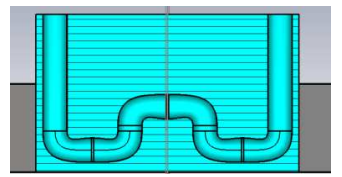

b)
Fig. 1. Side view of the a) U-pipe design b) W-pipe design. The chamber protrudes above the broad wall slot for access. The length of the insert in the horizontal direction $1=3.8 \mathrm{~mm}$, the height in the vertical direction $\mathrm{h}=2.27 \mathrm{~mm}$. The pipe diameter is $350 \mu \mathrm{m}$.

The transverse current at the centre of the broad wall is zero [11], whereas the $\mathrm{TE}_{10}$ mode wave electric field intensity inside is maximum at the centre [12]. A slot centred at this location would reduce interruption of this current. Simultaneously, it places the sample chamber in the high intensity region of the wave. The waveguide slot dimensions are $3.8 \times 0.6 \mathrm{~mm}$.

\section{SIMULATION}

For all simulations, a parametric sweep of the real permittivity, $\varepsilon_{r}^{\prime}$, of the fluid to be inserted inside the microfluidic pipe has been carried out using $1 \leq \varepsilon_{r}^{\prime} \leq 6$ in steps of 0.5 . The U-pipe is simulated with varying pipe depths, where the depth of the pipe is a fraction of the waveguide height, as can be seen in Fig. 2, where the depths are located 
at $3 / 4,1 / 2$, and $1 / 4$ of the waveguide height. The $\mathrm{W}$-pipe is simulated using only $1 / 2$ and $1 / 4$ the waveguide height are used, to avoid the middle of the pipe protruding above the waveguide slot.

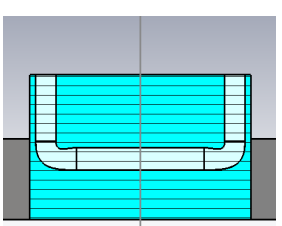

a)

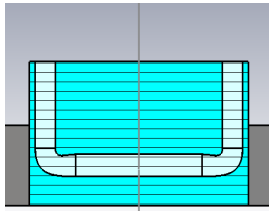

b)

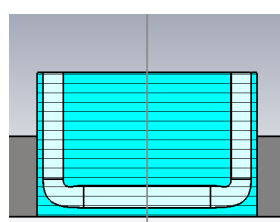

Fig. 2. A sliced view of the U-pipe insert, where the depth is given as a fraction of the waveguide height: a) $3 / 4$, b) $1 / 2$, and c) $1 / 4$

With the optimum depth for each pipe shape, the two configurations have $D_{p}$ swept, from 0.2 to $0.4 \mathrm{~mm}$ and 0.2 to $0.35 \mathrm{~mm}$ for the U-pipe and $\mathrm{W}$-pipe respectively, in steps of $0.05 \mathrm{~mm}$.

Further, the configurations with the most prominent effects with respect to $\varepsilon_{r}{ }^{\prime}$ will then be subjected to a parametric study. Where $\varepsilon_{r}{ }^{\prime}$ follows the sweep of the previous simulations, and $\tan \delta$ swept from 0 to 1 in steps of 0.1 .

\section{RESULTS}

In Fig. 3, $\left|\mathrm{S}_{11}\right|$ can be seen for the U-pipe and W-pipe. Each resonant curve shows a clear shift for a change in $\varepsilon_{r}^{\prime}$. Both the U-pipe and $\mathrm{W}$-pipe depths have a clear effect on the resonance spacing, lower depths result in a larger shift in frequency when $\varepsilon_{r}^{\prime}$ changes. A larger shift would distiguish better between two different permittivity values. In Fig. 4, a larger change in $\left|S_{21}\right|$ trace amplitude is observed as the pipe is positioned deeper. It is observed that with increasing $\varepsilon_{r}^{\prime}$, in the ideal lossless case, the drop in magnitude becomes increasingly larger. This change in magnitude is larger for pipes deeper inside the waveguide, for this reason the lowest pipe depth has been chosen for the later simulations.
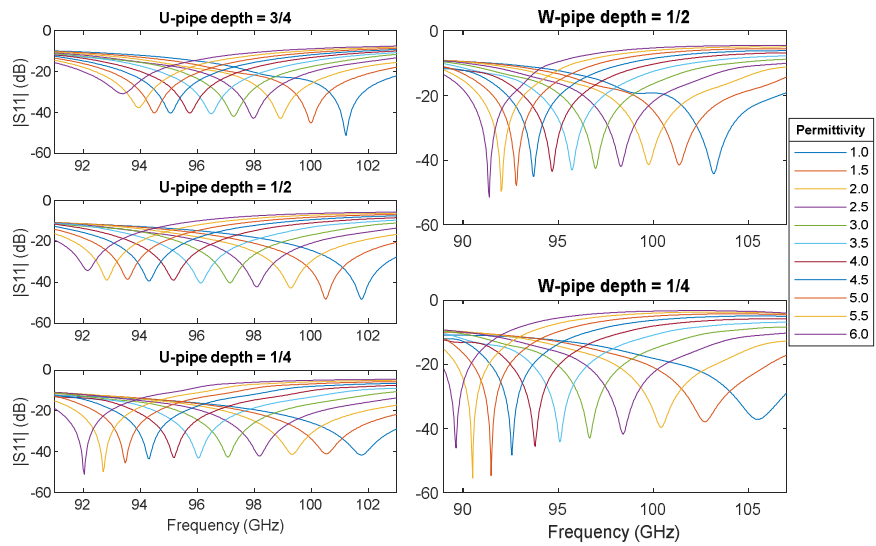

Fig. 3. $\mathrm{S}_{11}$ for the (left) U-pipe at the 3 varying depths inside the waveguide, and (right) W-pipe at 2 different depths. $\varepsilon_{r}^{\prime}$ is swept from 1 to 6 in steps of 0.5 .

Fig. 5 shows the resonant frequency vs $\varepsilon_{r}^{\prime}$, plotted for varying $D_{p}$, clearly a larger $D_{p}$ will lead to a larger shift in resonance for a change in $\varepsilon_{r}^{\prime}$. This is attributed to a larger volume of liquid interacting with the propagating wave.

Fig. 6 displays the spacing between traces for $\Delta\left|S_{21}\right|$, which becomes most apparent for the largest $D_{p}$. At values of $\varepsilon_{r}^{\prime} \leq 3.5$ spikes, rather than a smooth curve, are observed.
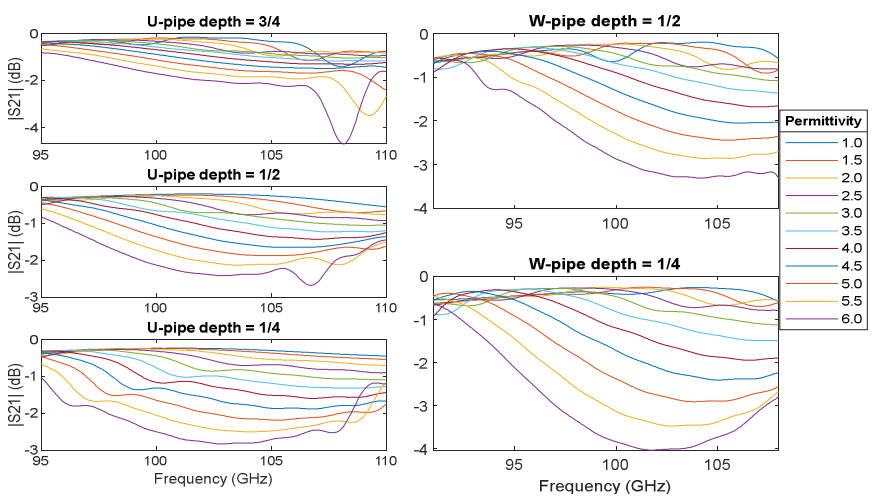

Fig. 4. $\mathrm{S}_{21}$ for the (left) U-pipe at the 3 varying depths inside the waveguide, and (right) W-pipe at 2 different depths. $\varepsilon_{r}^{\prime}$ is swept from 1 to 6 in steps of 0.5 .
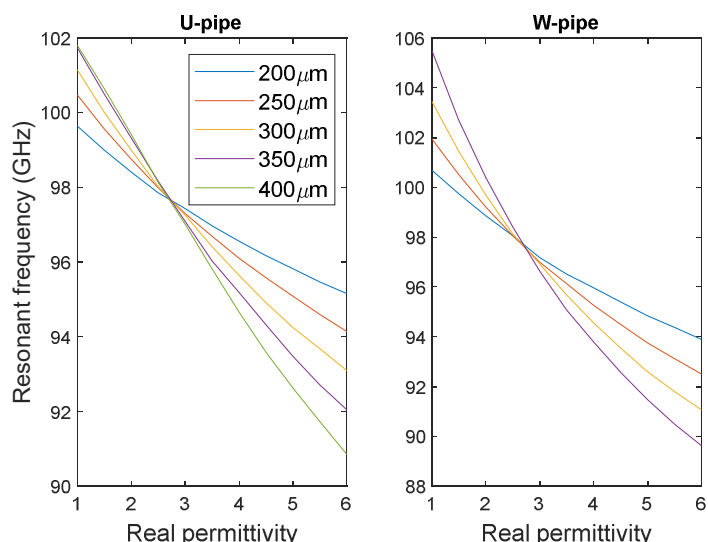

Fig. 5. The S11 resonance vs permittivity, for varying $D_{p}$. (left) U-pipe, (right) W-pipe. The legend applies to both graphs, except $400 \mu \mathrm{m}$ does not exist for the W-pipe.

These are due to ripples in $\left|\mathrm{S}_{21}\right|$ at these frequencies. These ripples can be seen at all depths for the U-pipe and $\mathrm{W}$-pipe in Fig. 4. The spacing between $\left|S_{21}\right|$ becomes larger with increasing $\varepsilon_{r}^{\prime}$. The largest $D_{p}$ for both pipes will be used for further simulations.
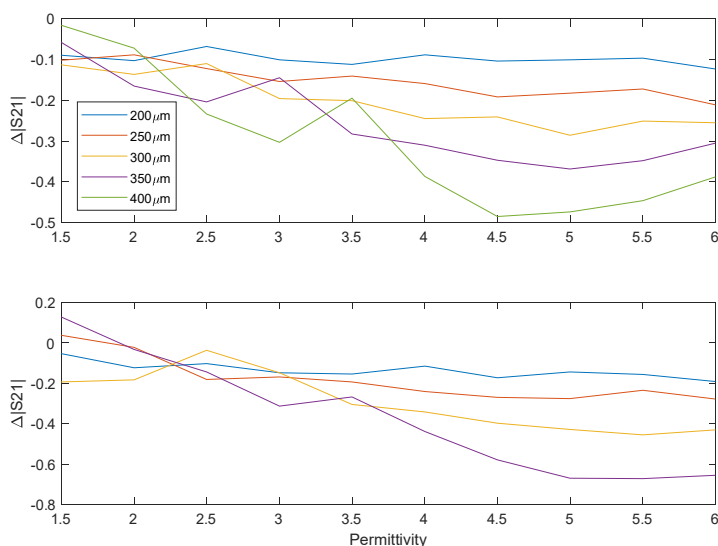

Fig. 6. Difference in $\left|\mathrm{S}_{21}\right|$ between permittivity traces for varying $D_{p}$ (top) U-pipe traces taken at frequencies 107.27, 105.59, 105.28, 104.16, and 102.51 GHz respectively for increasing $D_{p}$ (bottom) W-pipe at frequencies $101.57,106.19,104.58$, and $101.57 \mathrm{GHz}$ respectively.

The influence of increasing $\tan \delta$ on the $\left|\mathrm{S}_{11}\right|$ resonance frequency, for varying $\varepsilon_{r}^{\prime}$ is illustrated in 

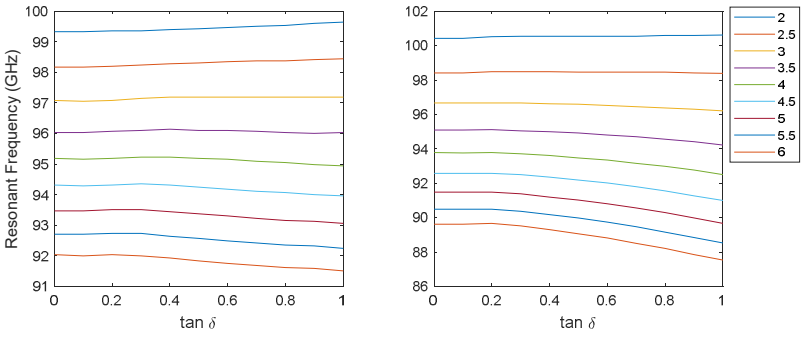

Fig. 7. Location of $|\mathrm{S} 11|$ resonance frequency vs $\tan \delta$ for varying $\varepsilon_{r}^{\prime}$. (left) U-pipe, (right) W-pipe.

Fig. 7, the shift is a result of smoothing of the resonance. $\varepsilon_{r}^{\prime} \geq$ 4 experience larger frequency shifts, and the $\mathrm{W}$-pipe is more sensitive to this effect than the U-pipe. In these configurations, volumes below the waveguide slot are: U-pipe $V=$ $0.4122 \mu l$, and W-pipe $V=0.4834 \mu l$, this difference in volume is a likely cause for this increased sensitivity.

Fig. 8 depicts the $\left|\mathrm{S}_{21}\right|$ traces for swept $\tan \delta$ from 0 to 1 , for both the $\mathrm{U}$ and $\mathrm{W}$-pipe at, $\varepsilon_{r}^{\prime}=2$ and 6 . Distinct and measurable shifts are present in $\left|\mathrm{S}_{21}\right|$ between traces for both the $U$ and $W$-pipe, at both values of $\varepsilon_{r}^{\prime}$.
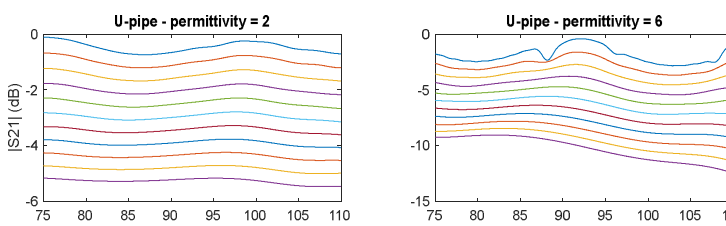

W-pipe - permittivity $=2$
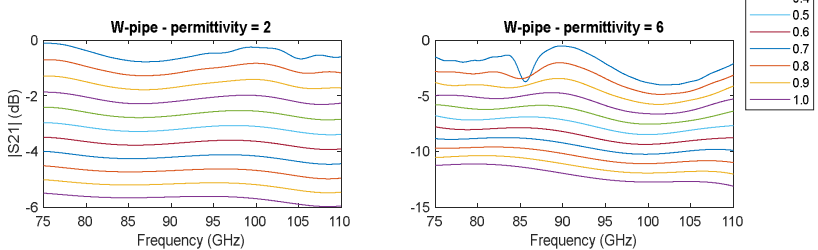

Fig. 8. $\left|\mathrm{S}_{21}\right|$ traces for both the U-pipe and W-pipe, $\tan \delta$ has been swept from 0 to 1 . The plots show $\varepsilon_{r}^{\prime}$ at values of 2 and 6 to show the comparison of the trace spacing.
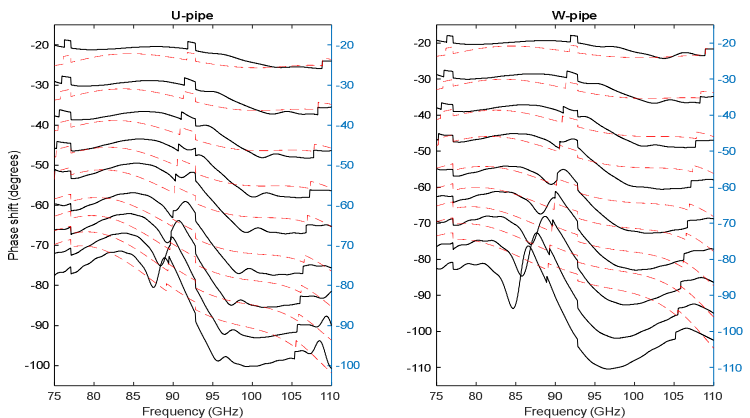

Fig. 9. The relative phase shift with respect to the air filled pipes. The black $\operatorname{traces} \operatorname{are} \tan \delta=0$, and the dashed red $\operatorname{traces} \tan \delta=1$. Looking at the highest frequency, from top to bottom the traces signify increasing $\varepsilon_{r}^{\prime}$ from 2 to 6 in steps of 0.5 .

In Fig. 9, the traces show phase shift relative to the unloaded fluid pipe. From top to bottom, the traces are for ascending permittivity from $\varepsilon_{r}^{\prime}=2$ to 6 with 0.5 steps. The black traces are $\tan \delta=0$, and the dashed red traces $\operatorname{are} \tan \delta=1$. The endpoint of a trace is the most stable point to determine the relative shift, this point varies the least with respect to permittivity and $\tan \delta$.

The current state of this work, shows promising results for enabling extraction of both $\varepsilon_{r}^{\prime}$ and $\tan \delta$. Comparing the $\mathrm{U}$ and W-pipe, with maximum dimensions, the U-pipe is the least affected by the $\left|S_{11}\right|$ resonance shift caused by varying $\tan \delta$. Hence providing less uncertainty in the analysis. This, alongside measurable shifts in $\mathrm{S}_{21}$ magnitude and phase, makes it currently the most suitable design for pairing all these parameters to determine both $\varepsilon_{r}^{\prime}$ and $\tan \delta$.

\section{CONCLUSIONS}

The presented design shows potential for extraction of the complex permittivity of fluids situated inside the microfluidic pipe. Where the response of each parameter can clearly be seen. The $\mathrm{S}_{11}$ minima is mostly linked to $\varepsilon_{r}^{\prime}$, with a smaller contribution from $\varepsilon_{r}^{\prime \prime}$. Whilst $\mathrm{S}_{21}$ amplitude is influenced by both $\varepsilon_{r}^{\prime}$ and $\varepsilon_{r}^{\prime \prime}$. Using empirical dependences obtained in this work, experimental results will be matched and the complex permittivity of the fluid under test extracted. For future work, the extraction process will use an ABCD matrix approach.

\section{ACKNOWLEDGMENT}

R. C. J. thanks the EPSRC for funding this research, grant No. EP/N50953X/1.

\section{REFERENCES}

[1] J. Qiu, B. Yang, O. Sushko, R. W. Pikersgill and R. S. Donnan, "Comparing Terahertz transmission response on $\mathrm{pH}$-dependent apomyoglobin proteins dynamics with circular dichroism," IMWSBio2014, 2014, pp. 1-3, doi: 10.1109/IMWS-BIO.2014.7032420.

[2] X. Liu, and J. Yu, "Characterization of the dielectric properties of water and methanol in the D-band using a quasi-optical spectroscopy," Sci. Rep. 9, 2019, pp. 18562

[3] F. Higgins, and A. Rein, "FTIR transmission measurement of volatile liquids using the Cary 630 FTIR equipped with DialPath technology," Application note [Online], May 19 1992. Available: https://www.agilent.com/cs/library/applications/59908540EN_AppNote_630_DialPath_Volatile_Liq.pdf

[4] E. Pucci, A. Zaman, and E. Rajo-Iglesias "Study of Q-Factors of Ridge and Groove Gap Waveguide Resonators," IET Microwaves, Antennas \& Propagation, 2013, vol. 7(11), pp. 900-908.

[5] E. N. Shaforost, N. Klein, S. A. Vitusevich, A. Offenhäusser, and A. A. Barannik, "Nanoliter liquid characterization by open whisperinggallery mode dielectric resonators at millimeter wave frequencies," $J$. Appl. Phys, 2008, vol. 104, pp. 074111.

[6] S. M. Hanham, C. Watts, W. J. Otter, S. Lucyszyn, and N. Klein, "Dielectric measurements of nanoliter liquids with a photonic crystal resonator at terahertz frequencies," Appl. Phys. Lett., 2015, vol. 107, pp. 032903

[7] E. McKeever, et al, "Label-free Chemical/Biochemical Sensing Device based on an Integrated Microfluidic Channel within a Waveguide Resonator" ESTC, 2010, pp. 1-3

[8] N. Chudpooti, E. Silavwe, P. Akkaraekthalin, I. D. Robertson and N. Somjit, "Nano-Fluidic Millimeter-Wave Lab-on-a-Waveguide Sensor for Liquid-Mixture Characterization," in IEEE Sensors Journal, vol. 18 , no. 1 , pp. $157-164$

[9] M. N. Afsar, N. Suwanvisan, and Y. Wang, "Permittivity measurement of low and high loss liquids in the frequency range of 8 to $40 \mathrm{GHz}$ using waveguide transmission line technique," Microw. Opt. Technol. Lett., vol. 48: pp. 275-281. https://doi.org/10.1002/mop.21326

[10] M. Schmid, D. Ludescher, and H. Giessen "Optical properties of photoresists for femtosecond 3D printing: refractive index, extinction, luminescence-dose dependence, aging, heat treatment and comparison between 1-photon and 2-photon exposure," Opt. Mater. Express, 2019, vol. 9(12), pp. 4564-4577

[11] R. A. Gilbert, "Waveguide Slot Antenna Arrays," in Antenna Engineering Handbook 4th ed. J. L. Volakis, USA: McGraw Hill Professional, 2007, ch. 9, pp. 9-2 - 9-5

[12] N. Marcuvitz , "Transmission-Line Modes" in Waveguide Handbook (IET Electromagnetic Waves Series 21). P. J. B. Clarricoats, E. D. R. Shearman, and J. R. Wait, Eds., UK: IET, 1986, ch. 2, pp. 55-6 